\title{
Multivariate scaling of maximum proton energy in intense laser driven ion acceleration
}

\author{
Yuji Takagi $\odot,{ }^{1,2, *}$ Natsumi Iwata $\odot,{ }^{2,3}$ Emmanuel d'Humieres, ${ }^{4}$ and Yasuhiko Sentoku $\odot^{2}$ \\ ${ }^{1}$ Department of Physics, Graduate School of Science, Osaka University, 1-1 Machikanecho, Toyonaka, Osaka 560-0043, Japan \\ ${ }^{2}$ Institute of Laser Engineering, Osaka University, 2-6 Yamadaoka, Suita, Osaka 565-0871, Japan \\ ${ }^{3}$ Institute for Advanced Co-Creation Studies, Osaka University, 1-1 Yamadaoka, Suita, Osaka 565-0871, Japan \\ ${ }^{4}$ CELIA, UMR No. 5107, CEA, CNRS, Université de Bordeaux, 351 Cours de la Libération 33400 Talence, France
}

(Received 26 July 2021; accepted 14 October 2021; published 29 November 2021)

\begin{abstract}
The production of high-energy ions is a momentous goal of ultraintense laser lights. So far a number of experiments and numerical simulations have been conducted to obtain the scaling of the ion energy to find the optimal experimental condition. Due to the complexity of the relativistic laser-plasma interactions, it is not easy to evaluate the ion energy for different experimental configurations. We propose a statistical approach using the Bayesian inference to obtain a multivariate scaling to predict the maximum proton energy via the target normal sheath acceleration. We derive the scaling for the experimental parameters and also for the hot electron temperature and density observed in the corresponding particle-in-cell simulations. We demonstrate the effectiveness of our approach in the prediction of the maximum proton energy and provide the experimental condition to achieve a proton energy over $100 \mathrm{MeV}$.
\end{abstract}

DOI: 10.1103/PhysRevResearch.3.043140

\section{INTRODUCTION}

The intensity of short pulse lasers has been increased to the level of $10^{21} \mathrm{~W} / \mathrm{cm}^{2}$ after the invention of chirped pulse amplification [1]. High-energy ion generation using such intense laser lights has been studied to realize compact ion sources for applications such as cancer therapy [2]. Several acceleration mechanisms of ions have been proposed, e.g., target normal sheath acceleration (TNSA) [3], collisionless shock acceleration, and radiation pressure acceleration. In TNSA, ions are accelerated by the sheath electric field generated at the rear side of a thin foil heated by intense laser light. Since TNSA is a controllable method that can produce ions with small emittance without being affected strongly by the plasma instabilities, TNSA has been studied intensively both experimentally [4-11] and theoretically [12-17].

There might be a true function for the maximum proton energy $E_{\max }$ accelerated by TNSA with variables such as laser intensity $I_{L}$, pulse length $\tau_{L}$, spot diameter $W$, and foil thickness $L$. If we know such a function, we will have a capability to provide experimental setups to achieve ion energies required in the applications, e.g., 100-MeV protons. The dependence of $E_{\max }$ on the experimental parameters has been studied with several approaches shown in Fig. 1. One approach is to use the regression analysis, i.e., a statistical approach to find the relation of the observed values with the experimental parameters as variables. So far, the analysis is

\footnotetext{
*takagi-y@ile.osaka-u.ac.jp

Published by the American Physical Society under the terms of the Creative Commons Attribution 4.0 International license. Further distribution of this work must maintain attribution to the author(s) and the published article's title, journal citation, and DOI.
}

limited to single variable scalings, e.g., the $E_{\max }$ dependence on $I_{L}$ [4] and the $E_{\max }$ dependence on $L[17,18]$.

Particle-in-cell (PIC) simulations have also been used for the prediction of $E_{\max }$. However, since not all the physics is included in the simulation models and also because the actual shot by shot conditions in the experiment such as preformed plasma distribution and laser intensity on the target are not clear, even three-dimensional PIC simulations cannot predict the experimental results accurately.

Recently, a neural-network-based approach, adapting 1000 one-dimensional (1D) PIC simulation data to train the network, has been used to forecast $E_{\max }$ for different plasma conditions, e.g., preformed plasma [19]. Theoretical models have been proposed with the support of the simulations. For instance, the isothermal plasma expansion theory [15] as well as employing the ponderomotive scaling [20] for the laseraccelerated hot electron temperature $T_{h}$ can describe well an ideal one-dimensional TNSA for short-pulse lasers qualitatively. However, as the theories use approximations, the quantitative prediction for $E_{\max }$ is difficult.

In this paper we propose a different approach, the multivariate regression analysis using Bayesian inference in combination with experimental data and also 1D PIC simulations, as illustrated in Fig. 1. The Bayesian inference was employed successfully to optimize the neutron yields in inertial confinement fusion [21].

In the past, the number of experiments using ultraintense laser light was limited due to the repeatability of the laser system. Thus, the experimental data covering a wide range of multidimensional parameter regimes were not available, so a multivariate analysis of experimental data had not been attempted. However, with the advent of high-repetition lasers in recent years, it is becoming possible to obtain a large amount of data with various experimental parameters. Our method proposed in this paper is considered to be an effective 


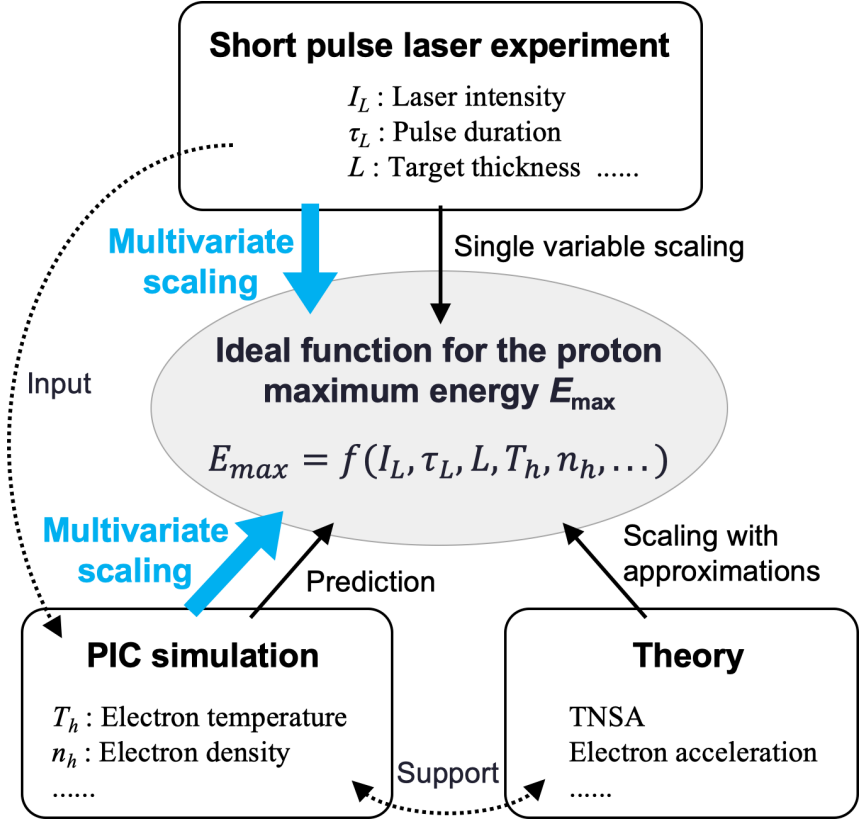

FIG. 1. Conventional approaches and the proposed approach (blue) for the prediction of the laser-driven ion acceleration.

tool to have a more accurate prediction of experiments in the future as more statistics will be available when more highrepetition-rate laser facilities will be commissioned. In this paper we assemble the previously published data [4-11] for the statistical analysis (see Fig. 2).

\section{CONVENTIONAL APPROACH FOR TNSA ENERGY PREDICTION}

Figure 2 shows the dependence of the proton maximum energy $E_{\max }$ on the laser intensity [Fig. 2(a)] and the pulse duration [Fig. 2(b)]. In Fig. 2(a) we plot two scalings for intensity, proportional to $I_{L}$ and proportional to $I_{L}^{1 / 2}$, with gray lines. From the data it is impossible to tell which scaling represents the $I_{L}$ dependence of $E_{\max }$. Beyond $10^{20} \mathrm{~W} / \mathrm{cm}^{2}$, $E_{\max }$ is saturated. There are two reasons for the saturation. One is the limitation of the focusing spot size, which should be larger than the excursion length $l_{\mathrm{ex}}$ of electrons [5]. The excursion length is the electron's oscillation length in the laser electric field and it is given as $l_{\mathrm{ex}}=a_{0} \lambda_{L} / 2 \pi$, where $\lambda_{L}$ is the laser wavelength, $a_{0}=e E_{L} / m_{e} c \omega_{L}$ the normalized amplitude of the laser field, $e$ the elementary charge, $E_{L}$ the laser electric field amplitude, $m_{e}$ the rest mass of the electron, $c$ the light speed, and $\omega_{L}$ the laser angular frequency. When the laser light is focused on a small spot to increase its intensity, $l_{\mathrm{ex}}$ eventually becomes larger than the laser spot size. The electrons then slip out from the interaction region before they get full acceleration, and thus the electron acceleration becomes inefficient.

The other reason is the pulse profile, especially the rising edge. In the case of an extremely intensified laser light over $10^{20} \mathrm{~W} / \mathrm{cm}^{2}$, the rising edge could have the relativistic intensity before the main pulse. The TNSA then starts before the main pulse arrives at the target [26]. Such a preacceleration can cause the detachment of protons from the target,
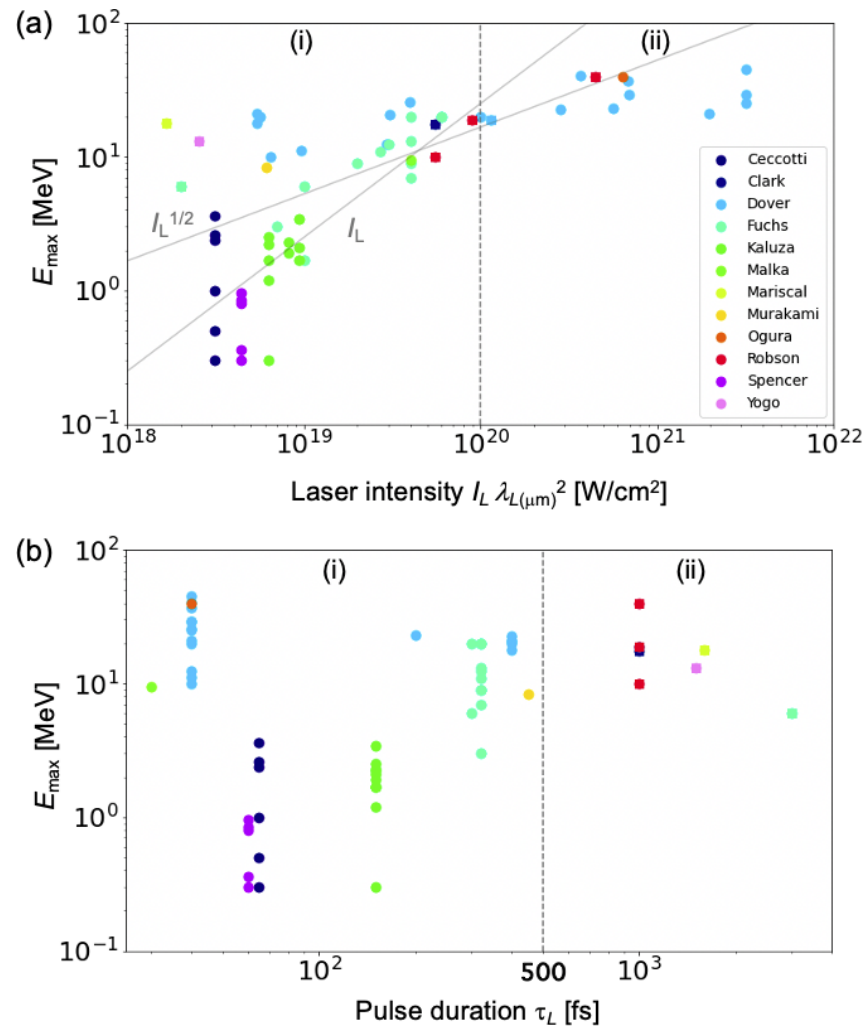

FIG. 2. Experimentally observed proton maximum energies against (a) laser intensity and (b) pulse duration. The data are taken from Ceccotti et al. [22], Clark et al. [23], Dover et al. [5], Fuchs et al. [4], Kaluza et al. [6], Malka et al. [11], Mariscal et al. [10], Murakami et al. [24], Ogura et al. [25], Robson et al. [9], Spencer et al. [7], and Yogo et al. [8].

preventing them from being accelerated by the main pulse. We thus discriminate the experimental data by the intensity (i) below and (ii) above $10^{20} \mathrm{~W} / \mathrm{cm}^{2}$.

Regarding the pulse duration, it has been reported that laser lights having pulse durations greater than 1 ps show a different property for the proton acceleration $[8,10]$, because of the change of the laser absorption due to the plasma blowout [27]. We therefore distinguish the data by the pulse duration (i) below and (ii) above 500 fs.

We perform the corresponding 1D PIC simulations with the PICLS code [28]. The foil target is modeled as a fully ionized plasma consisting of hydrogen and deuteron evenly. The initial target electron density is set to $253 n_{c}$. It is high enough density that the targets can survive during the laser plasma interaction even with the highest intensity. A preplasma is placed in front of the target. The preplasma starts from $50 n_{c}$ and drops exponentially with a $0.5-\mu \mathrm{m}$ scale length in $3 \mu \mathrm{m}$. We fixed the preplasma distribution for all the experimental conditions. The laser light has an amplitude $a_{0}$ with a flat intensity profile and rises and drops with the Gaussian shape with a 33-fs temporal width. We start the simulation two e-foldings down from the peak amplitude. The total pulse duration is set as the experimental one.

We plot $E_{\max }$ obtained in the simulations against that obtained in the corresponding experiment by the red plus points in Fig. 3. When the predicted $E_{\max }$ and the $E_{\max }$ in the 


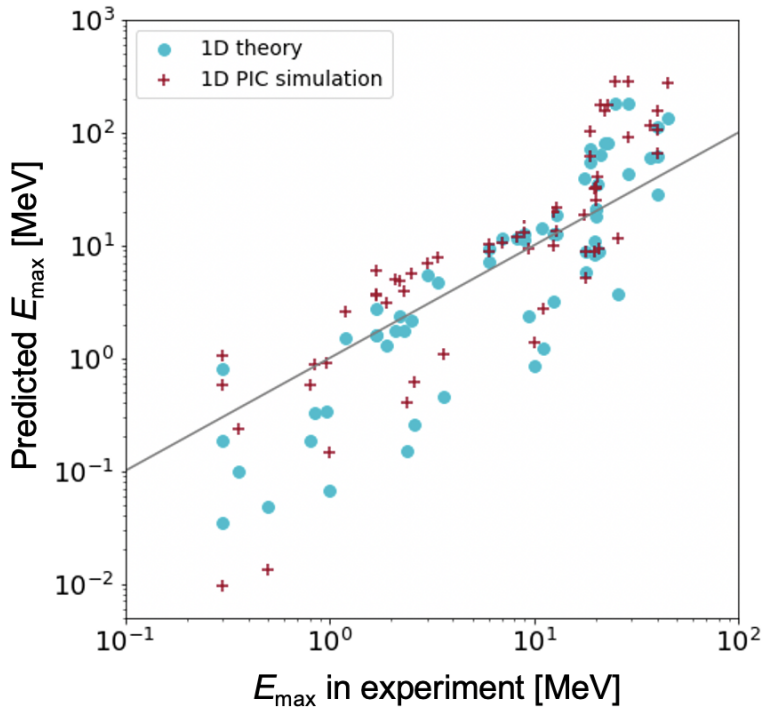

FIG. 3. Prediction for the maximum proton energy $E_{\max }$ obtained from the 1D PIC simulations (light blue points) and the 1D theoretical model [15] (red pluses). The solid line shows that the predicted $E_{\max }$ equals the $E_{\max }$ observed in the experiments.

experiment have the same value, the point is on the guiding solid line. The $E_{\max }$ in the simulations show higher values than the experimental ones for most of the cases. In other words, the 1D PIC overestimates the proton energy. In order to improve the accuracy, we need to have more precise information in the experiment such as the preformed plasma characteristic and we need to perform multidimensional PIC simulations. However, the quantitative prediction is still hard. We can adjust the maximum proton energy of the PIC simulation by changing, for example, the preformed plasma scale length, but not in a sophisticated way. Instead we will consider a rising edge of the incident laser light in the later analysis.

We also plot $E_{\max }$ predicted by a theoretical model by Mora [15], $E_{\max }=2 T_{h}\left\{\ln \left[\tilde{\tau}_{a}+\left(\tilde{\tau}_{a}^{2}+1\right)^{1 / 2}\right]\right\}^{2}$, by blue circles. Here $\tilde{\tau}_{a}$ is the normalized acceleration time $\tilde{\tau}_{a} \equiv \omega_{p i} \tau_{a} /\left(2 e_{N}\right)^{1 / 2}$, $\omega_{p i}=\left(4 \pi n_{h} e^{2} / M_{i}\right)^{1 / 2}$ is the ion plasma frequency of the proton at the hot electron density $n_{h}, M_{i}$ is the ion mass, and $e_{N}$ is Napier's constant. We use $T_{h}$ and $n_{h}$ evaluated in the simulations and set the acceleration time $\tau_{a}$ with the rule defined in Ref. [29], $\tau_{a} \equiv \kappa\left(\tau_{L}+t_{\min }\right)$, where $t_{\min } \equiv 60$ fs and $\kappa$ varies linearly from 3 at an intensity of $2 \times 10^{18} \mathrm{~W} / \mathrm{cm}^{2}$ to 1.3 at $3 \times 10^{19} \mathrm{~W} / \mathrm{cm}^{2}$ and stays constant at 1.3 for higher intensities. Note that $\kappa=1.3$ corresponds to the scaling in Ref. [4]. The theoretical values are scattered around the solid line, although they show a similar trend to the 1D PIC results, i.e., they overestimate the experimental values in the highintensity regime.

\section{STATISTICAL APPROACH FOR TNSA ENERGY PREDICTION}

The formula for predicting the maximum acceleration energy of protons was derived by regression analysis based on the framework of Bayesian inference. The experimental data $[4-11,22-25]$ in Fig. 2 have the parameter range for intensities $\left(1.5 \times 10^{18}\right)-\left(5 \times 10^{21}\right) \mathrm{W} / \mathrm{cm}^{2}$, pulse lengths $30-3000 \mathrm{fs}$, focal spots 2.6-60 $\mu \mathrm{m}$, and target thicknesses $0.5-125 \mu \mathrm{m}$.

We use the linear regression analysis based on the Bayesian statistics. The objective variable $\hat{y}_{i}$ has the average value

$$
\hat{y}_{i}=\sum_{k=0}^{K} \beta_{k} x_{k i}+\varepsilon_{i},
$$

where $i$ is the number of data of Fig. 2, $\beta_{k}$ are the coefficients of the linear regression, $x_{k i}$ are the explanatory variables, and $\varepsilon_{i}$ is the uncertainty having a normal distribution with the dispersion $\sigma^{2}$, i.e., $N\left(0, \sigma^{2}\right)$. In order to estimate the distribution of $\hat{y}_{i}$, we apply the Bayesian inference with the Markov chain Monte Carlo method using the PyMC3 library [30].

Here we assume the objective variable $\hat{y}_{i}=\ln \left(E_{\max i}\right)$ in the form

$$
\ln \left(E_{\max i}\right)=\sum_{k=0}^{K} \beta_{k} \ln \left(x_{k i}\right)+\varepsilon_{i},
$$

where $x_{k i}$ is the parameters in the simulations or experiments, except $x_{0 i}=\exp (1)$ for all $i$. We can rewrite Eq. (2) as

$$
E_{\max i}=\exp \left(\beta_{0}\right) \prod_{k=1}^{K} x_{k i}^{\beta_{k}} \exp \left(\varepsilon_{i}\right)
$$

which has the log-normal distribution. To obtain the indices $\beta_{k}$, we have to choose $x_{k}$ from the variables related to the TNSA.

First, we apply the Bayesian inference for the simulation results. In the following analyses, we take the initial distribution of each $\beta_{k}$ to be uniform. We use data in area (i) of Figs. 2(a) and 2(b) for the training and the validation. Since we know that the TNSA depends on the hot electron temperature $T_{h}$ and density $n_{h}$ from Mora's theory, we choose these two values in the 1D PIC simulations. The regression analysis yields

$$
E_{\max }(\mathrm{MeV})=7.77\left(\frac{T_{h}}{m_{e} c^{2}}\right)^{0.61}\left(\frac{n_{h}}{n_{c}}\right)^{0.31}\left(\frac{W}{10 \mu \mathrm{m}}\right)^{0.65} .
$$

In the derivation of Eq. (4), the dispersion of $\ln \left(E_{\max }\right)$ is $\sigma^{2}=0.34$. Equation (4) then has an uncertainty with a relative standard deviation of $\left[\exp \left(\sigma^{2}\right)-1\right]^{1 / 2} \exp \left(\sigma^{2} / 2\right)=0.75$. The coefficient of determination $R^{2}=1-(N-1) \sum_{i}\left(y_{i}-\right.$ $\left.\hat{y}_{i}\right)^{2} /(N-K-2) \sum_{i}\left(y_{i}-\bar{y}\right)^{2}$, which is a quick summary of the model fit in linear regressions, is 0.79 in this calculation, where $y_{i}$ is the observed value of $\hat{y}_{i}, \bar{y}$ is the averaged value of $y_{i}$, and $N$ is the total number of data. In Eq. (4) we also put the spot diameter $W$ as an explanatory variable to include the multidimensional effect, which is missing from 1D PIC simulations. The $E_{\max }$ in Eq. (4) depends strongly on $T_{h}$ as in the conventional understanding [15] and also has dependences on $n_{h}$ and $W$.

Figure 4 shows the prediction from Eq. (4). The black line indicates that the predicted $E_{\max }$ equals the experimental one. Both the training data (blue closed circles) and the validation data (orange closed circles) reproduce the experimental values well. Compared with Fig. 3, the prediction is improved notably, even though we only use the 1D simulations for the 


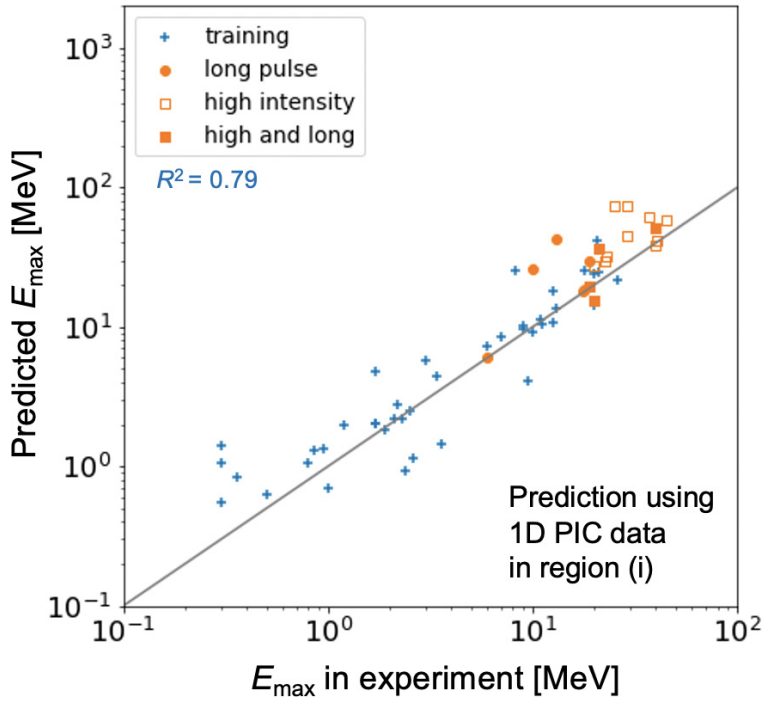

FIG. 4. Prediction for the maximum proton energy $E_{\max }$ by Eq. (4) for the Bayesian inference applied to the simulation parameters. The blue pluses are the training data in region (i). The orange closed circles, open squares, and closed squares are the data in region (ii) of Fig. 2 to see the applicability of the prediction to the parameter regime outside the training data. Here $R^{2}$ is the coefficient of determination.

prediction. Data shown by the light blue plots, which are from region (ii) in Fig. 2, are scattered around the black line.

We here check the dependences of $T_{h}$ and $n_{h}$ on the experimental parameters, intensity $I_{L}$, pulse duration $\tau_{L}$, and target areal mass density $\rho L$. The parameter $\rho L$ is introduced to take into account the stopping length of fast electrons. Using the same Bayesian inference, we obtain

$$
\begin{aligned}
& T_{h}(\mathrm{MeV}) \\
& =1.16\left(I_{20} \lambda_{\mu \mathrm{m}}^{2}\right)^{0.41}\left(\frac{\tau_{L}}{10 T_{L}}\right)^{0.07}\left(\frac{\rho}{\rho_{\mathrm{Al}}} \frac{L}{1 \mu \mathrm{m}}\right)^{-0.01}
\end{aligned}
$$

and

$$
\frac{n_{h}}{n_{c}}=38.4\left(I_{20} \lambda_{\mu \mathrm{m}}^{2}\right)^{1.47}\left(\frac{\tau_{L}}{10 T_{L}}\right)^{1.66}\left(\frac{\rho}{\rho_{\mathrm{Al}}} \frac{L}{1 \mu \mathrm{m}}\right)^{-1.03},
$$

where $I_{20}$ is the laser intensity normalized by $10^{20} \mathrm{~W} / \mathrm{cm}^{2}$, $\lambda_{\mu \mathrm{m}}$ is the laser wavelength normalized by $1 \mu \mathrm{m}, T_{L}=3.3$ fs is the oscillation period of a light with wavelength $1 \mu \mathrm{m}$, and $\rho_{\mathrm{Al}}$ is the mass density of aluminum. Interestingly, Eq. (5) shows that $T_{h}$ is proportional to $I_{L}^{1 / 2}$ and has almost no dependence on the other parameters. This $T_{h}$ scaling is consistent with the ponderomotive scaling [20]. Here $n_{h}$ depends on $I_{L}$, and also on $\tau_{L}$ and $1 / L$ as a result of the recirculation [18]. By substituting Eqs. (5) and (6) into Eq. (4), we find that $E_{\max }$ is almost proportional to the intensity $I_{L}$. The preplasma scale length $\ell_{p}$ set in the simulation mainly affects the scaling of $n_{h}$. The first coefficient on the right-hand side of Eq. (6) decreases almost linearly with $\ell_{p}$, by changing the scale length as $\ell_{p}=0.25,0.5$, and $0.75 \mu \mathrm{m}$. The coefficient and power indices in Eq. (4) change within the error range accordingly.

Next we apply the regression analysis to obtain the $E_{\max }$ scaling with respect to the experimental parameters $I_{L}, \tau_{L}, \rho L$,

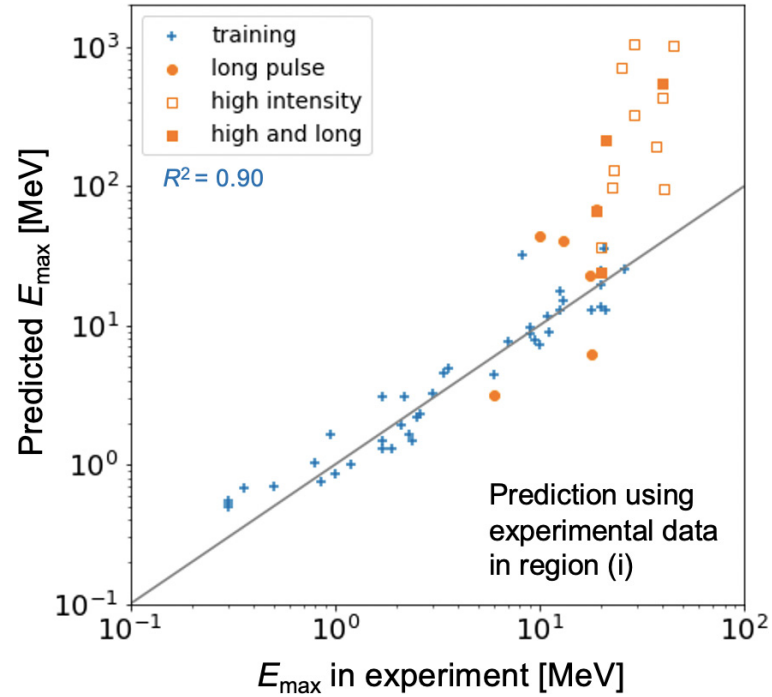

FIG. 5. Prediction for the maximum proton energy $E_{\max }$ by Eq. (7) for the Bayesian inference applied to the experimental parameters. The markers are the same manner as those in Fig. 4.

and $W$ from region (i) of Fig. 2 and obtain

$$
\begin{aligned}
E_{\max }(\mathrm{MeV})= & 132.8\left(I_{20} \lambda_{\mu \mathrm{m}}^{2}\right)^{0.95}\left(\frac{\tau_{L}}{10 T_{L}}\right)^{0.51} \\
& \times\left(\frac{\rho}{\rho_{\mathrm{Al}}} \frac{L}{1 \mu \mathrm{m}}\right)^{-0.62}\left(\frac{W}{10 \mu \mathrm{m}}\right)^{0.70},
\end{aligned}
$$

with an uncertainty with a relative standard deviation of 0.47 $\left(\sigma^{2}=0.17\right)$ and $R^{2}=0.90$. Equation (7) is consistent with the prediction using the simulations, $E_{\max } \propto I_{L}$, and the other parameter dependences. We plot the prediction by Eq. (7) in Fig. 5. The training and validation data are distributed along the black line. The data in region (ii) of Fig. 2, i.e., an intensity over $10^{20} \mathrm{~W} / \mathrm{cm}^{2}$ and a pulse duration over 500 fs, are overestimated. We thus cannot extrapolate $E_{\max }$ for the data in region (ii) using Eq. (7).

We now do the Bayesian inference including the data in the region of high intensity, (ii) of Fig. 2(a), to see the overall scaling. We have

$$
\begin{aligned}
E_{\max }(\mathrm{MeV})= & 27.1\left(I_{20} \lambda_{\mu \mathrm{m}}^{2}\right)^{0.61}\left(\frac{\tau_{L}}{10 T_{L}}\right)^{0.68} \\
& \times\left(\frac{\rho}{\rho_{\mathrm{Al}}} \frac{L}{1 \mu \mathrm{m}}\right)^{-0.43}\left(\frac{W}{10 \mu \mathrm{m}}\right)^{0.82},
\end{aligned}
$$

with an uncertainty with a relative standard deviation of 0.64 $\left(\sigma^{2}=0.27\right)$ and $R^{2}=0.86$. In Eq. (8) we see a weaker dependence on the intensity than in Eq. (7). The first coefficient is also reduced significantly to about $\frac{1}{5}$. Figure 6 shows the prediction by Eq. (8). All the data including those from the high intensities scatter around the black line. Note here that Eq. (8) indicates that lasers with an intensity of $10^{20} \mathrm{~W} / \mathrm{cm}^{2}$, a pulse duration of $100 \mathrm{fs}\left(\simeq 30 T_{L}\right)$, and a spot diameter of $10 \mu \mathrm{m}$ and $\mathrm{Al}$ targets with $1 \mu \mathrm{m}$ thickness produce only $E_{\max }=34.8 \mathrm{MeV}$. Such a low ion energy is attributable to the analysis including the low-performance experimental data over $10^{20} \mathrm{~W} / \mathrm{cm}^{2}$. 


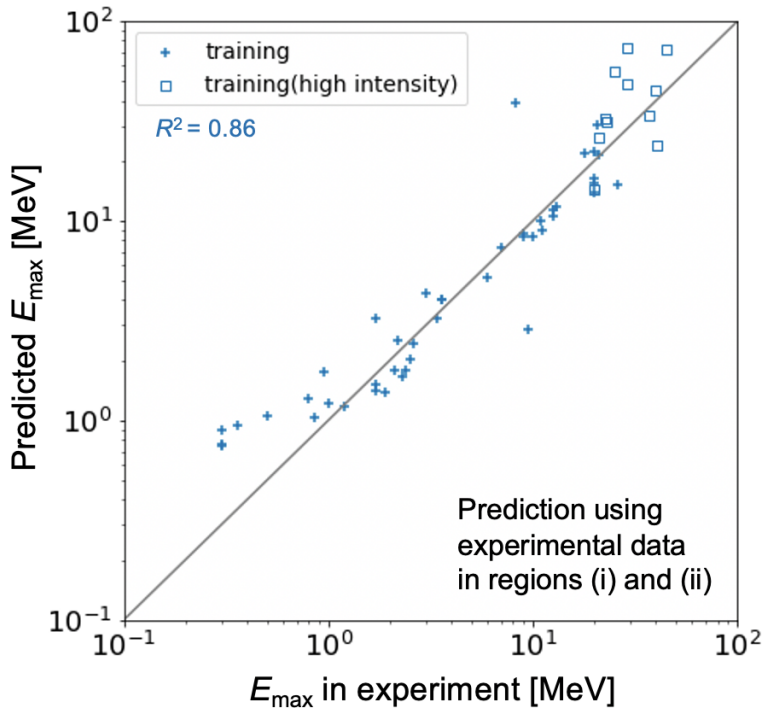

FIG. 6. The prediction for the maximum proton energy $E_{\max }$ by Eq. (8) including the data in the high intensity region (ii) of Fig. 2(a). The markers are the same as in Fig. 4.

There are two reasons for the low performance as we discussed above, i.e., tight focusing and an unavoidable rising edge of the high-intensity lasers. The relativistic rising edge preaccelerates protons in a contamination layer and makes them detach from the target; thus the main pulse could not accelerate the protons efficiently [26]. Hence the time when the rising edge exceeds the relativistic intensity, defined as $\tau_{\text {pre }}$, is an important parameter for the proton acceleration, especially for extremely intensified laser lights. We here model the rising edge by fitting the observed rising edge of the J-KAREN-P laser pulse [26] as a Gaussian with temporal width $\tau_{f}=250$ fs and having a peak intensity of $10^{20} \mathrm{~W} / \mathrm{cm}^{2}$ at the main pulse peak of $5 \times 10^{21} \mathrm{~W} / \mathrm{cm}^{2}$; thus the peak ratio (main pulse to rising edge) is 50 . The rising edge exceeds the relativistic intensity (greater than $10^{18} \mathrm{~W} / \mathrm{cm}^{2}$ ) at $\tau_{\text {pre }} \simeq 400$ fs before the main pulse with this profile. For the main peak intensity of $I_{L} \lambda_{\mu \mathrm{m}}^{2}<8 \times 10^{19} \mathrm{~W} / \mathrm{cm}^{2}$ we use $\tau_{\text {pre }}=\tau_{f} \arcsin \left(0.8 I_{20} \lambda_{\mu \mathrm{m}}^{2}\right)$ to avoid a sharp transition of $\tau_{\text {pre }}$. In the regression analysis, we keep the peak ratio 50 and scale the peak of the rising edge with the same pulse profile to estimate $\tau_{\text {pre. }}$. In order to include the above two effects, we introduce two additional explanatory variables $\left(\tau_{L}+\tau_{\text {pre }}\right) / \tau_{L}$ and $\left(W+2 l_{\text {ex }}\right) / W$ for the regression analysis and derive

$$
\begin{aligned}
E_{\max }(\mathrm{MeV})= & 152.1\left(I_{20} \lambda_{\mu \mathrm{m}}^{2}\right)^{1.01}\left(\frac{\tau_{L}}{10 T_{L}}\right)^{0.51} \\
& \times\left(\frac{\rho}{\rho_{\mathrm{Al}}} \frac{L}{1 \mu \mathrm{m}}\right)^{-0.56}\left(\frac{W}{10 \mu \mathrm{m}}\right)^{0.61} \\
& \times\left(\frac{\tau_{L}+\tau_{\mathrm{pre}}}{\tau_{L}}\right)^{-0.48}\left(\frac{W+2 l_{\mathrm{ex}}}{W}\right)^{-1.42},
\end{aligned}
$$

with an uncertainty with a relative standard deviation of 0.48 $\left(\sigma^{2}=0.18\right)$ and $R^{2}=0.91$. The power index for $I_{20} \lambda_{\mu \mathrm{m}}^{2}$ is recovered to 1.01 , which is close to that in Eq. (7), and the first coefficient exceeds $100 \mathrm{MeV}$. In addition, $\tau_{\text {pre }}$ for a peak intensity of $10^{20} \mathrm{~W} / \mathrm{cm}^{2}$ is about the pulse dura-

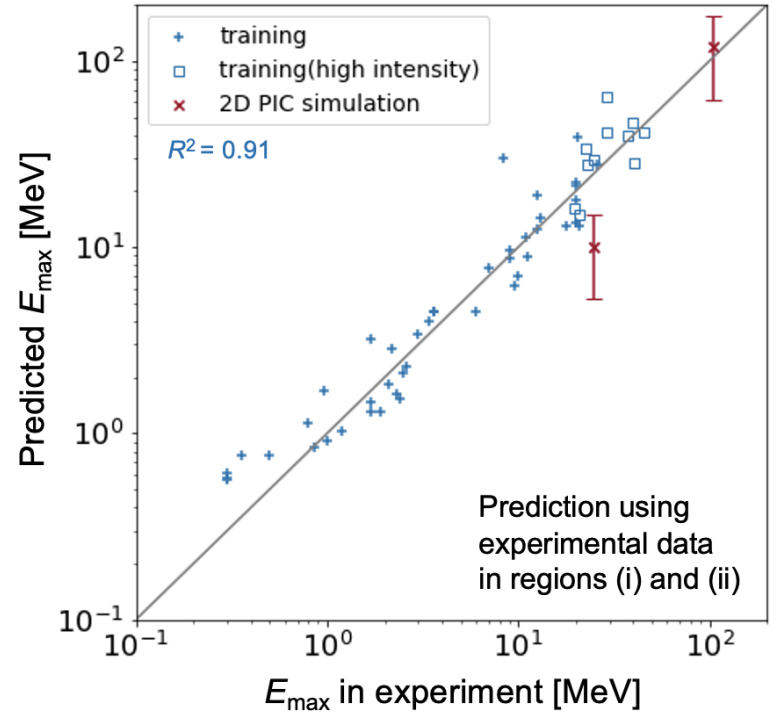

FIG. 7. Prediction for the maximum proton energy $E_{\max }$ by Eq. (9). The markers are the same as in Fig. 4. The high-intensity data in region (ii) of Fig. 2(a) are also used in the training. Red crosses are validations using 2D PIC simulations.

tion for high contrast lasers. Regarding the excursion length, $l_{\mathrm{ex}} \simeq 1.33 \mu \mathrm{m}$ for $I_{L}=1.5 \times 10^{20} \mathrm{~W} / \mathrm{cm}^{2}$ and $\lambda=0.8 \mu \mathrm{m}$, so $\left[\left(W+2 l_{\text {ex }}\right) / W\right]^{-1.42} \simeq 0.84$ for $W=10 \mu \mathrm{m}$, resulting in $E_{\max } \simeq 122 \mathrm{MeV}$ for $\tau_{L}=33 \mathrm{fs}\left(\simeq 10 T_{L}\right)$ and in $\mathrm{Al}$ targets with thickness $L=1 \mu \mathrm{m}$. We can make the energy even higher with targets thinner than $1 \mu \mathrm{m}$; however, another limitation is due to the target preheating by the prepulse as discussed in Ref. [6].

The predictions from Eq. (9) are shown in Fig. 7. The data points are scattered around the black line similarly to Fig. 6 . We here emphasize that the prediction by Eq. (9) agrees with physical understanding of the overall parameter dependences without losing the accuracy.

Figure 8 shows the maximum proton energy $E_{\max }$ predicted by Eq. (9) for a laser with $10 \mathrm{~J}$ by changing the pulse duration $\tau_{L}$ and laser intensity $I_{L}$. The laser spot diameter $W$ is determined automatically by $W=2 \sqrt{(10 \mathrm{~J}) / \pi I_{L} \tau_{L}}$. The $W$ cannot be smaller than the laser wavelength $\lambda_{L}$, which is the diffraction limit. This map shows what pulse configuration is favorable for the proton acceleration by TNSA. We see that the higher intensity results in a higher $E_{\max }$ unless the intensity exceeds about $10^{21} \mathrm{~W} / \mathrm{cm}^{2}$ due to the excursion length effect. The longer pulse duration can achieve a higher $E_{\max }$ than the shorter one can. This is because the rising edge effect becomes weaker.

We performed 2D PIC simulations using the PICLS code, which includes atomic processes such as Coulomb collisions and ionizations [31], for two different cases to benchmark the prediction of Eq. (9). The first case, case A, has a $2-\mu \mathrm{m}$ titanium foil irradiated by a J-KAREN-P laser light with an intensity of $3 \times 10^{21} \mathrm{~W} / \mathrm{cm}^{2}$, a $0.8-\mu \mathrm{m}$ wavelength, a spot diameter of $1.6 \mu \mathrm{m}$, and a $40-\mathrm{fs}$ pulse duration, including the relativistic rising edge $300 \mathrm{fs}$ before the main pulse. The second case, case $\mathrm{B}$, is the recommended configuration from Eq. (9), which has $1-\mu \mathrm{m}$ aluminum irradiated by a widely 


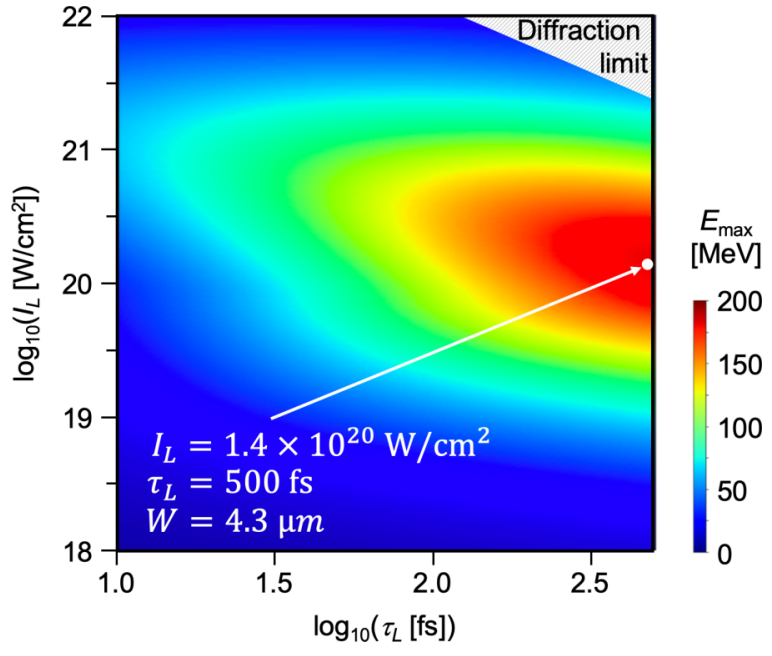

FIG. 8. Maximum proton energy $E_{\max }$ predicted by Eq. (9) for $10 \mathrm{~J}$ laser energy. The target is fixed to $1 \mu \mathrm{m}$ aluminum and the laser wavelength is $1 \mu \mathrm{m}$. The top right corner is an unreachable regime due to the diffraction limit, i.e., $W<\lambda_{L}$.

focused laser light with a lower intensity of $1.4 \times 10^{20}$ $\mathrm{W} / \mathrm{cm}^{2}$, a $1.0-\mu \mathrm{m}$ wavelength, a diameter of $12 \mu \mathrm{m}$, and a 40-fs pulse duration. The pulse has no relativistic rising edge but it exceeds the relativistic intensity 50 fs before the pulse peak. The pulse profiles used in the 2D PIC simulations are illustrated in Fig. 9. The target materials and thicknesses are different between cases A and B. We choose a heavier target in case $\mathrm{A}$ to ensure that the target is not distorted under highintensity laser irradiation. Both cases have a $20-\mathrm{nm}$ proton layer behind the target and a $2-\mu \mathrm{m}$ preformed plasma in front of the target.

Figure 10 shows the temporal evolution of energy densities of ions and protons in each case. We use different contour

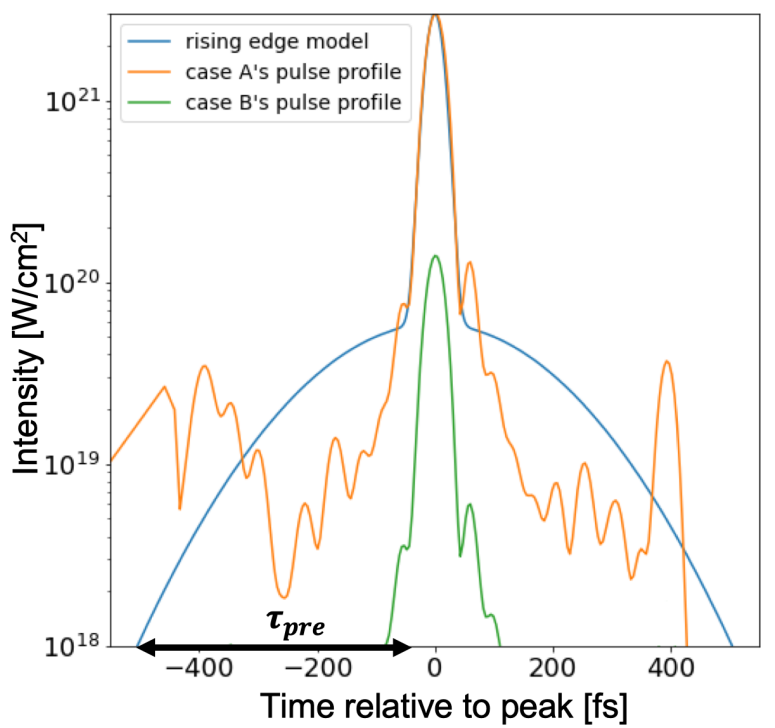

FIG. 9. Laser temporal profiles used in the 2D PIC simulations for cases A (orange) and B (green). An orange line is the temporal profile of a J-KAREN-P laser experiment (detailed in Ref. [26]). A blue line is the typical pulse profile used in the model in deriving Eq. (9).
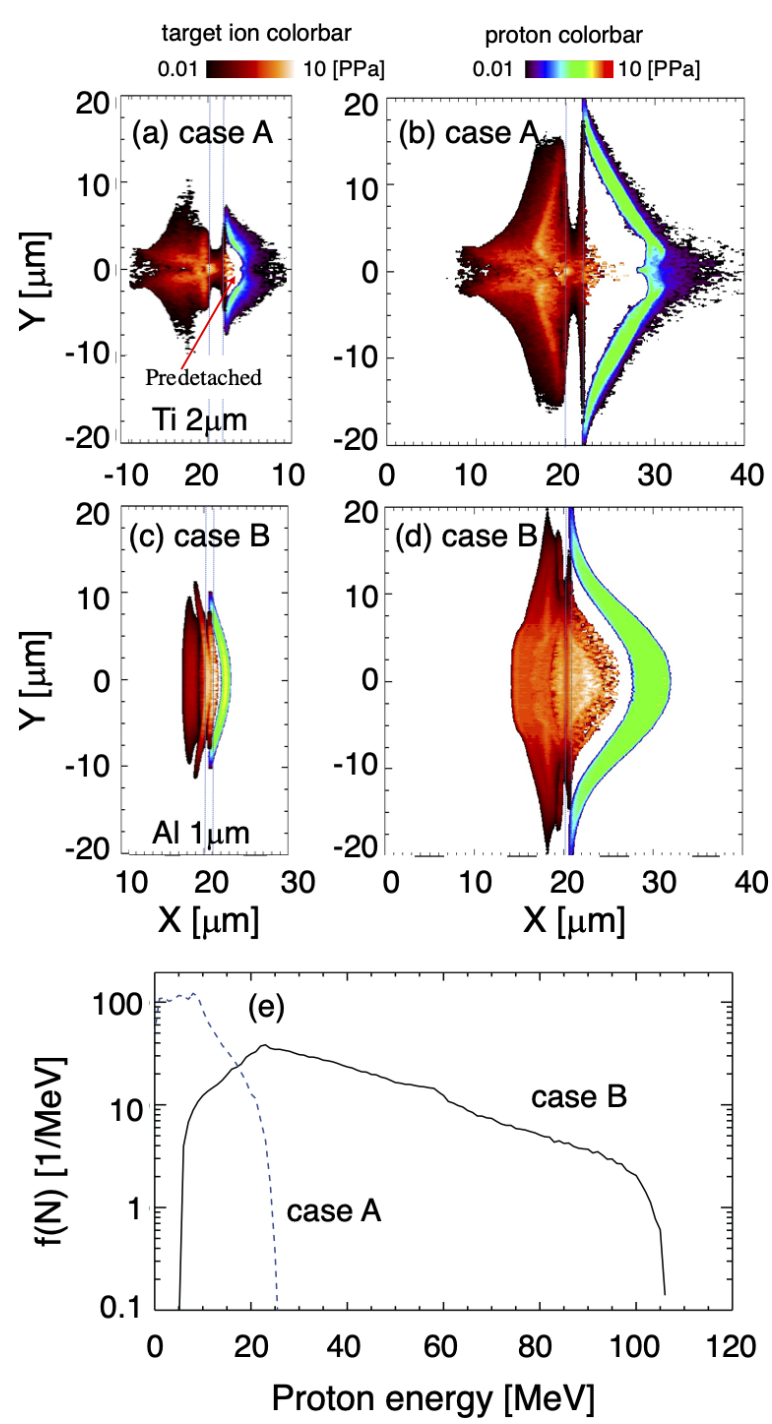

FIG. 10. 2D PIC simulation for a tightly focused laser pulse (case A) and a widely focused laser pulse (case B). Energy densities (in petapascal) of target ions (black, red, and white) and protons (rainbow) are shown for case A observed (a) right after the main pulse irradiation and (b) 100 fs later. (c) and (d) Same energy densities for case B, respectively. (e) Energy spectrum of protons for case A (dashed line) and case B (solid line)

colors for bulk ions and protons to distinguish them. In case A with the relativistic rising edge, the proton layer was predetached when the main pulse arrived. There is a vacuum gap behind the protons; thus hot electrons were stopped in the gap and they are not delivered to the proton front. As a result, the protons did not get efficiently accelerated but the bulk ions did, as discussed in Ref. [26]. In contrast, case B with a widely focused but lower peak intensity could achieve the standard TNSA without having negative impacts from the tight focusing and from the relativistic rising edge. The protons in case B [Fig. 10(d)] are continuously accelerated to energies greater than $100 \mathrm{MeV}$. We plot the simulation result with the predicted proton energy for each case with a red cross in Fig. 7.

Equation (9) predicts the proton maximum energy for case A, $10 \pm 4.9 \mathrm{MeV}$, and for case B, $119 \pm 57 \mathrm{MeV}$. Note here 
that case A has a proton maximum energy of $25 \mathrm{MeV}$, which is higher than the prediction. Since we used the 2D PIC simulation, the proton acceleration via TNSA in the tight focus laser plasma interaction is overestimated. In contrast, case B, with wider focus laser, demonstrates proton energy greater than $100 \mathrm{MeV}$, as predicted by Eq. (9).

\section{DISCUSSION AND CONCLUSION}

The scaling given by Eq. (9) tells us how to exceed 100 $\mathrm{MeV}$ in the TNSA of protons. Equation (9) shows that $E_{\max }$ is proportional to the laser intensity. Further, $E_{\max } \geqslant 100 \mathrm{MeV}$ requires laser intensities higher than $10^{20} \mathrm{~W} / \mathrm{cm}^{2}$. However, we must keep a wide focal spot to avoid the degradation of the electron acceleration. Pulse shape control is also essential to reduce the effect of the relativistic prepulse. The scaling derived here is for TNSA proton acceleration. A thinner target is better to produce higher $E_{\max }$ from Eq. (9), but when the target is too thin to be relativistically transparent, the acceleration mechanism shifts to other accelerations, e.g., radiation pressure acceleration, for which the scaling is not applicable.

In this paper we applied the Bayesian inference to obtain the scaling of the proton maximum energy via the TNSA, for which the physics is relatively well known. As we demonstrated the scaling of $T_{h}$ and $n_{h}$ [Eqs. (5) and (6)], this method is also a robust tool to find unknown dependences of physical quantities on experimental parameters. The same multivariate scaling approach will be beneficial to optimize other products in the relativistic laser-plasma interactions such as $\mathrm{x}$ and $\gamma$ rays and neutrons.

The neural-network-based modeling [19] might obtain a scaling equation but with a limited number of variables. Compared to the neural-network-based modeling, our approach has the advantage of providing a multivariate scaling with implementation of physical considerations, e.g., the effect of the excursion length, and thus is powerful tool to see a dependence on each variable for the objective, e.g., $E_{\max }$, of experimental setups.

Our scaling has an uncertainty of $\sigma^{2}$ in the Bayesian inference. This uncertainty could be smaller by increasing the training data. More current and near future laser systems have a high repetition rate and thus they are preferable for use in our approach.

\section{ACKNOWLEDGMENTS}

This study was supported by the Foundation for the Promotion of Ion Engineering and JSPS KAKENHI Grants No. JP19KK0072, No. JP20K14439, and No. JP20H00140. The authors thank Dr. M. Nishiuchi and Dr. N. Dover for providing J-KAREN-P data for the analysis and fruitful discussions.
[1] D. Strickland and G. Mourou, Compression of amplified chirped optical pulses, Opt. Commun. 56, 219 (1985).

[2] S. V. Bulanov, J. J. Wilkens, T. Z. Esirkepov, G. Korn, G. Kraft, S. Kraft, M. Molls, and V. S. Khoroshkov, Laser ion acceleration for hadron therapy, Phys. Usp. 57, 1149 (2014).

[3] A. Macchi, M. Borghesi, and M. Passoni, Ion acceleration by superintense laser-plasma interaction, Rev. Mod. Phys. 85, 751 (2013).

[4] J. Fuchs, P. Antici, E. D’Humieres, E. Lefebvre, M. Borghesi, E. Brambrink et al., Laser-driven proton scaling laws and new paths towards energy increase, Nat. Phys. 2, 48 (2006).

[5] N. P. Dover, M. Nishiuchi, H. Sakaki, K. Kondo, M. A. Alkhimova, A. Y. Faenov, M. Hata, N. Iwata, H. Kiriyama, J. K. Koga, T. Miyahara, T. A. Pikuz, A. S. Pirozhkov, A. Sagisaka, Y. Sentoku, Y. Watanabe, M. Kando, and K. Kondo, Effect of Small Focus on Electron Heating and Proton Acceleration in Ultrarelativistic Laser-Solid Interactions, Phys. Rev. Lett. 124, 084802 (2020).

[6] M. Kaluza, J. Schreiber, M. I. K. Santala, G. D. Tsakiris, K. Eidmann, J. Meyer-ter-Vehn, and K. J. Witte, Influence of the Laser Prepulse on Proton Acceleration in Thin-Foil Experiments, Phys. Rev. Lett. 93, 045003 (2004).

[7] I. Spencer, K. W. D. Ledingham, P. McKenna, T. McCanny, R. P. Singhal, P. S. Foster et al., Experimental study of proton emission from 60-fs, 200-mJ high-repetition-rate tabletop-laser pulses interacting with solid targets, Phys. Rev. E 67, 046402 (2003).

[8] A. Yogo, K. Mima, N. Iwata, S. Tosaki, A. Morace, Y. Arikawa et al., Boosting laser-ion acceleration with multi-picosecond pulses, Sci. Rep. 7, 42451 (2017).
[9] L. Robson, P. T. Simpson, R. J. Clarke, K. W. D. Ledinghan, F. Lindau, O. Lundh et al., Scaling of proton acceleration driven by petawatt-laser-plasma interactions, Nat. Phys. 3, 58 (2007).

[10] D. Mariscal, T. Ma, S. C. Wilks, A. J. Kemp, G. J. Williams, P. Michel et al., First demonstration of ARC-accelerated proton beams at the National Ignition Facility, Phys. Plasmas 26 , 043110 (2019).

[11] V. Malka, S. Fritzler, E. Lefebvre, E. d'Humieres, R. Ferrand, G. Grillon et al., Practicability of protontherapy using compact laser systems, Med. Phys. 31, 1587 (2004).

[12] J. E. Crow, P. L. Auer, and J. E. Allen, The expansion of a plasma into a vacuum, J. Plasma Phys. 14, 65 (1975).

[13] J. Denavit, Collisionless plasma expansion into a vacuum, Phys. Fluids 22, 1384 (1979).

[14] P. Mora and R. Pellat, Self-similar expansion of a plasma into a vacuum, Phys. Fluids 22, 2300 (1979).

[15] P. Mora, Plasma Expansion into a Vacuum, Phys. Rev. Lett. 90, 185002 (2003).

[16] V. T. Tikhonchuk, Physics of laser-assisted ion acceleration, Nucl. Instrum. Methods Phys. Res. Sect. A 620, 1 (2010).

[17] Y. Sentoku, T. E. Cowan, A. Kemp, and H. Ruhl, High energy proton acceleration in interaction of short laser pulse with dense plasma target, Phys. Plasmas 10, 2009 (2003).

[18] A. J. Mackinnon, Y. Sentoku, P. K. Patel, D. W. Price, S. Hatchett, M. H. Key, C. Andersen, R. Snavely, and R. R. Freeman, Enhancement of Proton Acceleration by Hot-Electron Recirculation in Thin Foils Irradiated by Ultraintense Laser Pulses, Phys. Rev. Lett. 88, 215006 (2002).

[19] B. Z. Djordjevic, A. J. Kemp, J. Kim, R. A. Simpson, S. C. Wilks, T. Ma, and D. A. Mariscal, Modeling laser-driven ion 
acceleration with deep learning, Phys. Plasmas 28, 043105 (2021).

[20] S. C. Wilks, W. L. Kruer, M. Tabak, and A. B. Langdon, Absorption of Ultra-Intense Laser Pulses, Phys. Rev. Lett. 69, 1383 (1992).

[21] V. Gopalaswamy, R. Betti, J. P. Knauer, N. Luciani, D. Patel, K. M. Woo et al., Tripled yield in direct-drive laser fusion through statistical modelling, Nature (London) 565, 581 (2019).

[22] T. Ceccotti, A. Levy, H. Popescu, F. Reau, P. D’Oliveira, P. Monot, J. P. Geindre, E. Lefebvre, and P. Martin, Proton Acceleration with High-Intensity Ultrahigh-Contrast Laser Pulses, Phys. Rev. Lett. 99, 185002 (2007).

[23] E. L. Clark, K. Krushelnick, J. R. Davies, M. Zepf, M. Tatarakis, F. N. Beg et al., Measurements of Energetic Proton Transport through Magnetized Plasma from Intense Laser Interactions with Solids, Phys. Rev. Lett. 84, 670 (2000).

[24] Y. Murakami, Y. Kitagawa, Y. Sentoku, M. Mori, R. Kodama, K. A. Tanaka, K. Mima, and T. Yamanaka, Observation of proton rear emission and possible gigagauss scale magnetic fields from ultra-intense laser illuminated plastic target, Phys. Plasmas 8, 4138 (2001).

[25] K. Ogura, M. Nishiuchi, A. S. Pirozhkov, T. Tanimoto, A. Sagisaka, T. Z. Esirkepov et al., Proton acceleration to $40 \mathrm{MeV}$ using a high intensity, high contrast optical parametric chirped- pulse amplification/Ti:sapphire hybrid laser system, Opt. Lett. 37, 2868 (2012).

[26] M. Nishiuchi, N. P. Dover, M. Hata, H. Sakaki, Ko. Kondo, H. F. Lowe, T. Miyahara, H. Kiriyama, J. K. Koga, N. Iwata et al., Dynamics of laser-driven heavy-ion acceleration clarified by ion charge states, Phys. Rev. Research 2, 033081 (2020).

[27] N. Iwata, S. Kojima, Y. Sentoku, M. Hata, and K. Mima, Plasma density limits for hole boring by intense laser pulses, Nat. Commun. 9, 623 (2018).

[28] Y. Sentoku and A. J. Kemp, Numerical methods for particle simulations at extreme densities and temperatures: Weighted particles, relativistic collisions and reduced currents, J. Comput. Phys. 227, 6846 (2008).

[29] J. Fuchs, Y. Sentoku, E. d'Humieres, T. E. Cowan, J. Cobble, P. Audebert et al., Comparative spectra and efficiencies of ions laser-accelerated forward from the front and rear surfaces of thin solid foils, Phys. Plasmas 14, 053105 (2007).

[30] J. Salvatier, T. V. Wiecki, and C. Fonnesbeck, Probabilistic programming in Python using PyMC3, PeerJ Comput. Sci. 2, e55 (2016).

[31] R. Mishra, P. Leblanc, Y. Sentoku, M. S. Wei, and F. N. Beg, Collisional particle-in-cell modeling for energy transport accompanied by atomic processes in dense plasmas, Phys. Plasmas 20, 072704 (2013). 